\title{
Online Calibration of a Humanoid Robot Head from Relative Encoders, IMU Readings and Visual Data
}

\author{
N. Moutinho, M. Brandão, R. Ferreira, J. Gaspar, A. Bernardino, J. Santos-Victor \\ Institute for Systems and Robotics / Instituto Superior Técnico \\ Lisbon, Portugal \\ \{nmoutinho, mbrandao, ricardo, jag, alex, jasv\}@isr.ist.utl.pt
}

\begin{abstract}
Humanoid robots are complex sensorimotor systems where the existence of internal models are of utmost importance both for control purposes and for predicting the changes in the world arising from the system's own actions. This so-called expected perception relies on the existence of accurate internal models of the robot's sensorimotor chains.

We assume that the kinematic model is known in advance but that the absolute offsets of the different axes cannot be directly retrieved from encoders. We propose a method to estimate such parameters, the zero position of the joints of a humanoid robotic head, by relying on proprioceptive sensors such as relative encoders and inertial sensing as well as visual input.

We show that our method can estimate the correct offsets of the different joints (i.e. absolute positioning) in a continuous, online manner. Not only the method is robust to noise but it can as well cope with and adjust to abrupt changes in the parameters. Experiments with three different robotic heads are presented and illustrate the performance of the methodology as well as the advantages of using such an approach.
\end{abstract}

\section{INTRODUCTION}

The application of robotic technologies in fields such as military, industry, service or research has grown quite significantly over the past years. As a consequence, the need for these robots to perform a large number of specific tasks has led to an impressive increase in their level of complexity and sophisticated control and perceptual modalities. Regardless of the concrete application domain, the availability of internal, properly calibrated, sensorimotor models is an ubiquitous and fundamental requirement.

Such internal models are instrumental for the control of such complex robots, as they allow to predict the consequences of the robot's own movements in a certain physical scenario. Perception can thus contrast predicted signals with actually observed measurements. This mechanism of $e x$ pected perception is believed to be used routinely by humans when addressing a variety of tasks including locomotion, grasping, manipulation, etc [1], [2], [3].

It is however important to note that the increased complexity of such robot systems, in particular the large number of degrees of freedom and sophisticated sensing, makes the calibration process quite challenging. Indeed, the calibration can be prohibitively time consuming and subject to errors,

This work has been partially supported by the EU Project RoboSoM, FP7ICT-248366 and by the Portuguese Science Foundation project DCCAL, PTDC / EEACRO / 105413 / 2008.

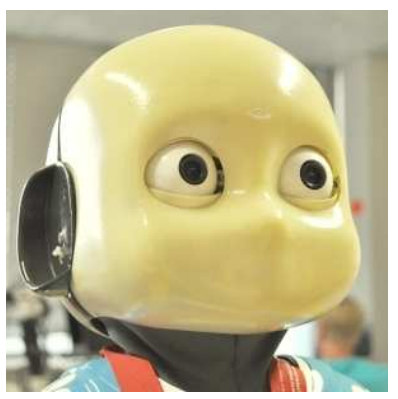

(a) iCub head

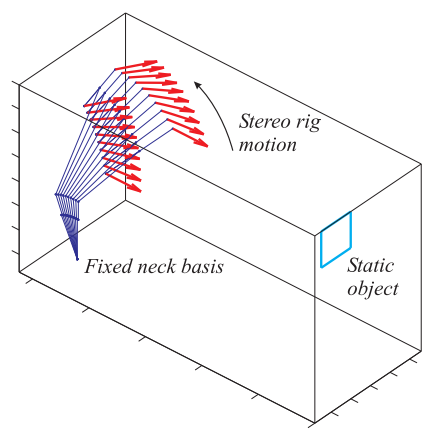

(c) Sample cameras motion

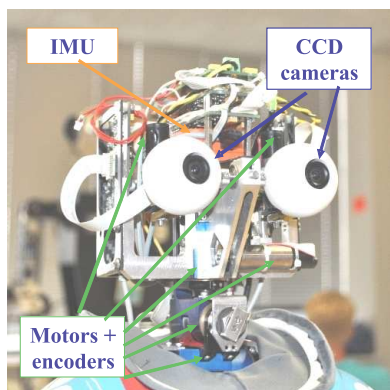

(b) Actuators and Sensors

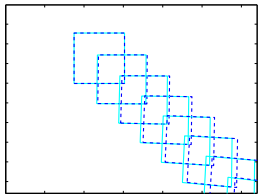

(d) $2^{o}$ offsets

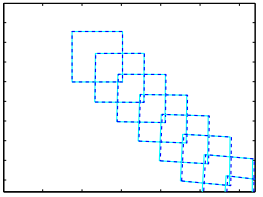

(e) $0.5^{\circ}$ offsets
Fig. 1. The iCub head used in this work (a). Sensors and actuators in the iCub head encompass two cameras, six motors and encoders, and one IMU (b). Example of a trajectory of the stereo cameras (red arrows), that observe an object in the scene (cyan square), to demonstrate the effect of the offsets in the expected perception (c). Images of the cyan square seen by the left camera and expected perception (dashed lines) when all the joints have $2^{\circ}$ offsets (d). Expected images of the cyan square when the joints have just $0.5^{\circ}$ offsets (e).

even if manually performed by experts. An alternative way, that we adopt in this work, is to exploit the robot's own embedded sensors and design automated calibration methods which are faster and more accurate than manual tuning, able to adjust to dynamic changes over time.

While the discussion is valid for many different systems, in this paper we focus on the specific case of humanoid robot heads. The heads are usually equipped with inertial sensors (IMU) and motor encoders that provide information concerning the rotation of the joints. This type of sensors can describe the movements of the robot, which is useful for calibration purposes, in terms of sensorimotor constraints. Since encoders provide relative measurements only, the absolute 
zero position of the head is normally assigned to the initial position when the system is turned on. The angular offsets of the joints need to be estimated in order to determine the system's real configuration at startup and ensure repeatability of operation.

The importance of having accurate internal models of the robot's sensorimotor chains has been stressed in a recent review [4]. Several works have addressed the problem of calibrating sensorimotor chains, by combining information provided by embedded or external sensors, like the IMU or cameras, with readings from encoders, to estimate the absolute offsets required for calibration. In [5] the authors present an algorithm that enables a humanoid robot to learn its kinematic function by visually observing its end-effectors when moving them. However this algorithm assumes an artificial form of measuring the information of the joints using specific known objects attached to the end-effector that needs to be always in the field of view of the cameras. A similar approach is presented in [6] where specific visual markers are used to learn the kinematic function of the robot. This algorithm finds the optimal set of robot configurations and observations that improve the learning process thus minimizing the required amount of data. Other works do not need specific markers or known objects to calibrate the robotic kinematic model, using restrictions in the signals sensors produced by the movements of the robot joints. The work described in [7] proposes a mathematical solution and implements a vision based method for the automatic calibration of a small pan-tilt system. This method is able to find the joints initial angles by tracking visual features in the images provided by a camera mounted at the end of the kinematic chain and by calculating the homography induced by rotations of the tilt joint. However, the authors point out that the estimation of the offsets of the joints depends largely on the quality of the tracker, since this system does not include filtering to attenuate the bad influence of such measurements. A different calibration method is presented in [8], to automatically calibrate the neck joints of a humanoid robot using measurements from the IMU. The approach uses the algorithm described in [9] to obtain - in real time - the values of the joints that allow the correct calibration of the head. However, like the previous work, this system does not include any type of noise filtering or disturbance rejection.

Ultimately, a good calibration result is always hard to obtain since it depends on the quality of the sensors, on the presence of noise or non-linearities such as joint backlash, where the encoders can no longer provide information about the actual rotation. As most robots are equipped with relative encoders, calibration of the absolute positions has to be done at startup time as well as during operation. A robust method is then required to provide the best results under any conditions.

Our contribution is thus an approach for online calibration of a real humanoid robotic head, in terms of its joints angles, and taking the kinematic model into account. The method proposed operates in a continuous manner, it is robust to noise and uses information from the embedded IMU, visual
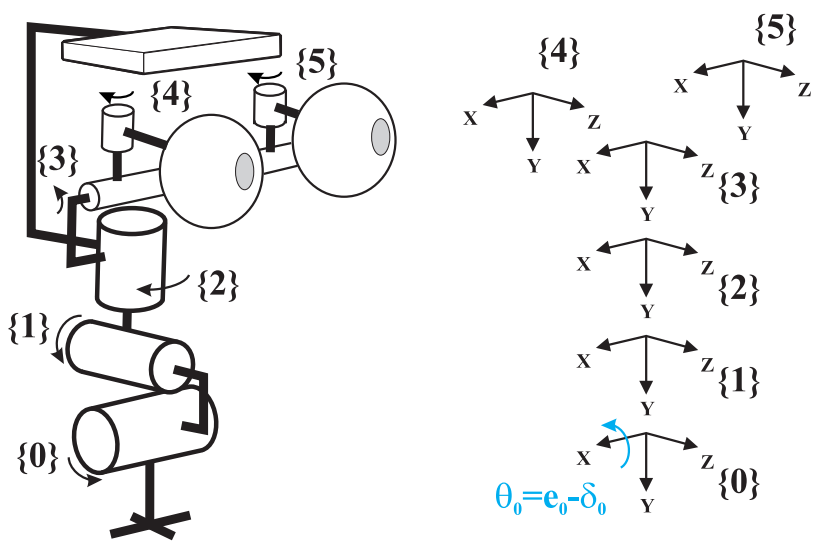

Fig. 2. iCub head main frames. The IMU (box on the top) is rigidly attached to frame $\{3\}$. Joint angles, $\theta_{i}$ are obtained from the encoder readings, $e_{i}$, after subtracting the offsets, i.e. zero position encoder readings $\delta_{i}$.

data the cameras and the relative measurements from the encoders, in the presence of noise and other disturbances. This system does not use any type of specific markers or known objects in the world or attached to the robot body.

The structure of the paper is as follows. Section II does an introduction to the calibration problem. Section III describes the methodology we propose for the calibration of a robotic head. Results obtained with different robotic head platforms are shown in section IV and conclusions are drawn in section V.

\section{PROBLEM Formulation}

It is common for humanoid robots to be equipped with encoder sensors which lack the capability of providing an absolute encoder position with respect to some factory calibrated value. These encoders fix their zero value at the position in which they are turned on, consequently needing to be calibrated each time the system is powered on.

We define the head absolute zero position as having the cameras looking forward, with parallel projection planes orthogonal to the floor, and a chosen gravity vector reading given by the IMU, usually corresponding to a perfectly vertical gravity vector pointing down. These sensors are commonly placed on top of the head thus not influenced by eye movement. For these sensor readings, we define the zero position of the reference frames as having the axis orientations $x$ pointing to the right, $y$ pointing down and $z$ pointing to the front (see figure 2).

The complete rotation ${ }^{i} R_{0}$ of a certain reference frame $i$ relative to the base of the kinematic chain 0 can be represented as a composition of elementary rotations around each joint. Considering that the joints are numbered sequentially, the kinematic model from the base frame $\{0\}$ to frame $\{n\}$ is written as

$$
{ }^{n} R_{0}\left(\theta_{0} \ldots \theta_{n}\right)={ }^{n} R_{n-1}\left(\theta_{n-1}\right) \ldots{ }^{2} R_{1}\left(\theta_{1}\right){ }^{1} R_{0}\left(\theta_{0}\right)
$$

where the different $\theta_{i}$ and ${ }^{i+1} R_{i}$ represent the angle and the one axis rotation function of each individual joint. This kinematic model can predict the measurements of each 
sensor since their spatial orientation in each time instant is known. In this work we assume the base of the kinematic chain is static and aligned with gravity in a planar horizontal surface, the world is static and infinite (all the objects seen by the cameras are at a very large distance) and there are no mounting errors of the sensors.

Due to the referred encoder resetting when powered up, the joint angles $\theta_{i}$ are given by the encoder readings $e_{i}$ subtracted by a constant $\delta_{i}$ which needs to be estimated. The objective of this work is to estimate the offsets $\delta_{i}$ such that the relation

$$
\theta_{i}=e_{i}-\delta_{i}
$$

holds. Collecting the joint offsets $\delta_{i}$ in a vector $\chi_{\Delta}$, the system state $\chi$ which is to be estimated at each time step is:

$$
\chi=\left[\chi_{G}, \chi_{\Delta}^{T}\right]^{T}
$$

where $\chi_{G}$ denotes the norm of the gravity, also to be estimated online in order to compensate offsets in the accelerometer readings.

\section{Calibration Methodology}

The base of our system is an Implicit Kalman Filter [10], which, given the state transition and the sensor observation equations is able to update the state in order to reduce the measurement error, thus providing the best estimates for the joints offsets.

\section{A. State Transition Model}

In our system state $\chi$ both the gravity norm as well as the joint offsets are assumed to be constant over time, thus the state transition equation $\mathcal{F}$ simply propagates the previous values. To allow for small changes of the values over time, e.g. due to mechanical wear or slippage, we allow for some state transition noise $w^{k}$.

The system state transition model $\mathcal{F}$ is therefore simply:

$$
\chi^{k \mid k-1}=\chi^{k-1}+w^{k}
$$

Here $w^{k} \sim N\left(0, Q^{k}\right)$ where $Q^{k}$ represents the covariance matrix of the zero mean state transition noise $w^{k}$. The system can be adapted to be more or less sensitive to variations in the estimate of the offsets by changing this covariance matrix.

\section{B. Observation Model}

In this work we are considering robotic platforms equipped with three types of sensors: IMU, cameras and encoders. In order to describe the observation model, we first introduce the notation for the measurements that we take from each of the sensors.

The IMU provides measurements at time $k$ for linear accelerations

$$
Z_{A}^{k}=\left[\begin{array}{lll}
a_{x}^{k} & a_{y}^{k} & a_{z}^{k}
\end{array}\right]
$$

and angular velocities

$$
Z_{W}^{k}=\left[\begin{array}{lll}
w_{x}^{k} & w_{y}^{k} & w_{z}^{k}
\end{array}\right]
$$

for the three principal axis of the frame that the IMU is rigidly attached to. We assume that the linear accelerations measured by the IMU correspond to the effects of the gravity vector decomposed in the three components of $x, y$ and $z$ affected by sensor noise.

The cameras provide $M$ image features represented by their image coordinates $f_{i}=\left[\begin{array}{ll}u_{i} & v_{i}\end{array}\right]$ which can be collected in the feature measurement vector at an instant $k$

$$
Z_{F}^{k}=\left[\begin{array}{lll}
f_{0}^{k} & \ldots & f_{M-1}^{k}
\end{array}\right] .
$$

We are interested in image movement induced by joint movement hence we always consider a pair of consecutive frames as measurements (for example $Z_{F}^{k}$ and $Z_{F}^{k-1}$ ). The features are assumed to be matched between the two time instants which is accomplished by applying the Harris Corner Detector [11] to image $k-1$ and then tracking the obtained features in image $k$ using an image patch to perform Normalized Cross Correlation within a given area.

Considering $N$ kinematic joints, a scan of all the encoders, $Z_{E}^{k}$ consists of $N$ measurements of the relative position of the joints taken at time instants $k$,

$$
Z_{E}^{k}=\left[\begin{array}{lll}
e_{0}^{k} & \ldots & e_{N-1}^{k}
\end{array}\right] .
$$

The encoders are sampled at the same instants as the other measurements, IMU or cameras.

Since the IMU seldomly works at the same frequency as the image acquisition sensors, these readings are usually not simultaneously available. Hence at each time step we either have an IMU observation or an image observation which needs to be filtered. The system measurements $Z^{k}$ are thus given by one of two possibilities at each time step $k$ :

$$
Z^{k}=\left[\begin{array}{llll}
Z_{A}^{k} & Z_{W}^{k} & Z_{E}^{k} & Z_{E}^{k-1}
\end{array}\right]+v_{I}^{k}
$$

or

$$
Z^{k}=\left[\begin{array}{llll}
Z_{F}^{k} & Z_{F}^{k-1} & Z_{E}^{k} & Z_{E}^{k-1}
\end{array}\right]+v_{C}^{k}
$$

where $v_{I}^{k} \sim N\left(0, R_{I}^{k}\right)$ and $v_{C}^{k} \sim N\left(0, R_{C}^{k}\right)$ are the observation noise in case of IMU or image measurements respectively, assumed to be a zero mean Gaussian with covariance matrix $R_{I}^{k}$ or $R_{C}^{k}$. These measurements provide physical constraints which are used by the filter to improve the state estimates.

In order to predict the sensor measurements the absolute value of each joint is needed to compute the complete rotations ${ }^{i} R_{0}$ from the base of the kinematic chain to the reference frame where each sensor is mounted. Collecting equations 2 in vector form, the absolute values of the joints $\Theta$ are given by

$$
\Theta^{k}=Z_{E}^{k}-\chi_{\Delta}^{k}
$$

Considering that the IMU is mounted on reference frame $I$ we represent the base to IMU coordinate transformation by ${ }^{I} R_{0}(\Theta)$. The linear accelerations measurement prediction $\hat{Z}_{A}$ are obtained by mapping the world constant gravity vector by this rotation:

$$
\hat{Z}_{A}\left(\chi^{k}, Z_{E}^{k}\right)={ }^{I} R_{0}\left(\Theta^{k}\right) \cdot\left[\begin{array}{lll}
0 & -\chi_{G}^{k} & 0
\end{array}\right]^{T}
$$


where $\chi_{G}^{k}$ is the prediction of the gravity norm present in the state vector $\chi$. Note the negative sign since the accelerometer measures gravitational reaction.

The angular velocities measurement predictions $\hat{Z}_{W}$ are computed from the derivative of the IMU reference frame, here approximated by the change of this reference frame between two consecutive time instants divided by the change in time. Since the base of the robotic platform does not move these velocities can be obtained from

$$
\hat{Z}_{W}^{k}\left(\chi^{k}, Z_{E}^{k}, Z_{E}^{k-1}\right)=\frac{\log \left({ }^{I} R_{0}\left(\Theta^{k}\right) \cdot{ }^{I} R_{0}^{-1}\left(\Theta^{k-1}\right)\right)}{d T}
$$

where the $\log$ function is implemented as the inverse Rodrigues function providing the instant angular change ${ }^{1}$ and $d T$ is the time interval between the two encoder measurements.

When a camera is available, mounted in reference frame $C$, the position of a set of previously observed features can be predicted assuming the world is infinite (image features only affected by rotation). The prediction of the $i$ th image feature $f_{i}$ is given by

$$
\hat{f}_{i}^{k}=P\left({ }^{C} R_{0}^{k} \cdot\left({ }^{C} R_{0}^{k-1}\right)^{-1} \cdot\left[\begin{array}{ll}
f_{i}^{k-1} & 1
\end{array}\right]^{T}\right)
$$

where $P$ is a function that divides the $x$ and $y$ components by $z$. This equation provides a set of constraints, two for each image feature, which can be collected as

$$
\hat{Z}_{F}\left(\chi^{k}, Z_{E}^{k}, Z_{E}^{k-1}, Z_{F}^{k-1}\right)=\left[\begin{array}{lll}
\hat{f}_{0}^{k} & \ldots & \hat{f}_{M-1}^{k}
\end{array}\right]^{T} .
$$

Thus, depending on the set of measurements available at a given time step (either equation 9 or 10) the observation function $\mathcal{H}^{k}$ of the implicit Kalman filter is either

$$
\mathcal{H}^{k}\left(\chi^{k}, Z^{k}\right)=\left[\begin{array}{c}
Z_{A}^{k}-\hat{Z}_{A}^{k}\left(\chi^{k}, Z_{E}^{k}\right) \\
Z_{W}^{k}-\hat{Z}_{W}^{k}\left(\chi^{k}, Z_{E}^{k}, Z_{E}^{k-1}\right)
\end{array}\right]=0
$$

or

$$
\mathcal{H}^{k}\left(\chi^{k}, Z^{k}\right)=Z_{F}^{k}-\hat{Z}_{F}\left(\chi^{k}, Z_{E}^{k}, Z_{E}^{k-1}, Z_{F}^{k-1}\right)=0 .
$$

By using this function as the filter's innovation, the system is able to correctly estimate all the joint offsets. As previously mentioned, an Implicit Kalman Filter is used since none of the measurement equations can be written in explicit form, defining instead a constraint that the measurements, together with the state need to satisfy.

\section{Results}

This section describes the experiments realized to test the behavior and performance of the proposed online calibration system. The experiments involved three humanoid heads / robots: the iCub robotic head at IST/UTL University [12], the Kobian robot at Waseda University [13] (see Figure 6(a)), and the Vizzy robot also at IST/UTL University (see Figure 6(b)).

The testing of multiple robots allows verifying the adaptability of the proposed system to different robotic platforms,

\footnotetext{
${ }^{1}$ The inverse Rodrigues formula provides a closed form solution to the Lie logarithm function on the rotation Lie group.
}

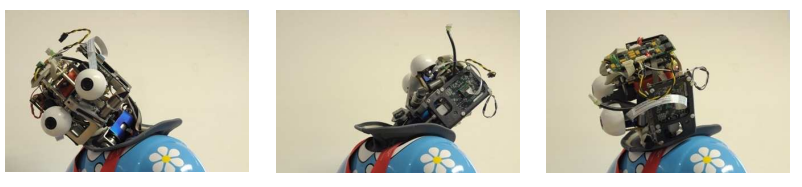

(a) Sample random positions.

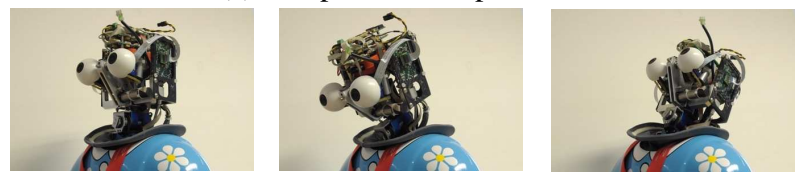

(b) Vertical, front and back positions.

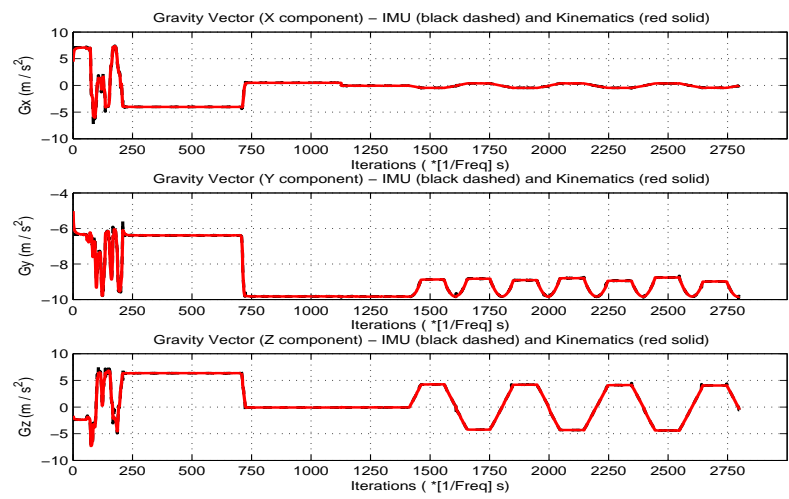

(c) Gravity observed (IMU, black) and estimated (red).

Fig. 3. A visual description of the calibration process for the iCub head A sequence of positions acquired during the random movement of the head (a). The calibrated position of the head completely vertical and the response of the head to a rectangular signal on the tilt motor (b). After converging the calibration, the head is successfully commanded to a vertical position, $G_{x}=0$ and $G_{z}=0$, and correctly shows a leaning forward and backwards pattern, $G_{x}=0$ and $G_{z}$ alternates max./min., corresponding to the rectangular signal applied to the tilt motor (c).

in particular having different schema and numbers of joints. The kinematic model of the iCub head encompasses six rotational joints, namely tilt, swing, pan, eyes tilt, left eye pan and right eye pan (see Figure 2). The Kobian robot is a humanoid platform with seven rotational joints in the head, namely swing, tilt, pan, tilt, eyes tilt, left eye pan and right eye pan. The Vizzy robot has also six rotational joints in the head, but in a different configuration (order) from the iCub head, namely tilt, pan, tilt, eyes tilt, left eye pan and right eye pan.

The calibration procedure common to all experiments, consists of initializing the head in an arbitrary uncalibrated position and rotating it randomly, either by hand or by motor control, as shown in figure 3 for the case of the iCub.

Two specific sets of experiments were realized with the iCub head (i) testing the absolute positioning error as read by the gravity measurements, and (ii) testing the repeatability of the calibration results obtained. The standard deviations of the observation noises were measured in order to have an accurate characterization of the sensors. The measured values are shown in table I along with the frequency associated to each sensor. 
TABLE I

CHARACTERIZATION OF THE SENSORS

\begin{tabular}{|c|c|c|}
\hline Sensor & Noise Std. Dev. & Frequency $(\mathrm{Hz})$ \\
\hline IMU (Linear Acceleration) & $0.22 \mathrm{~m} / \mathrm{s}^{2}$ & 10 \\
IMU (Angular Velocity) & $0.10 \mathrm{rad} / \mathrm{s}$ & 10 \\
Cameras & $3 \mathrm{pixel}$ & 30 \\
Encoders & $0.0005 \mathrm{rad}$ & $>30$ \\
\hline
\end{tabular}

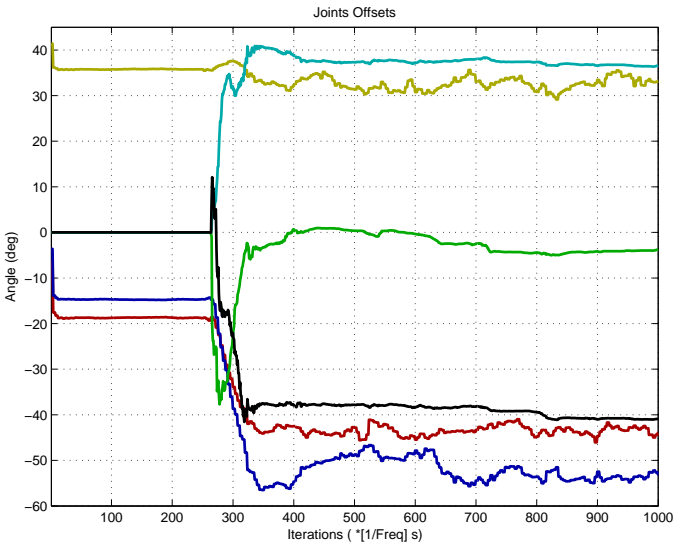

Fig. 4. Calibration of the iCub joints offsets - joint 0 (red), joint 1 (yellow), joint 2 (blue), joint 3 (green), joint 4 (purple) and joint 5 (black).

\section{A. Offset estimation with homing to the zero position}

To test and analyze the convergence of the system and the homing to the zero position we used the iCub robotic head as previously described. Figure 4 shows the convergence of the system to the offsets of the joints in approximately 70 iterations or 2.3 seconds, starting at iteration 260, corresponding to the moment the head was first moved. The filter was initialized with all offsets set to zero and it is worth noting that immediately after the first iteration the system has already assimilated the first reading of the accelerometer (the first 260 iterations in the figure). Since different head configurations can provide the same accelerometer readings (e.g. different pan angles with a fully upright head), this initial measurement is not enough to converge to the final configuration nor does it provide any information about the eye joints. The head movements are required to disambiguate these multiple solutions and provide the final offsets values.

As seen in the figure, the first three joints never fully converge to a single value, oscillating inside an interval $\left(\left[-46^{\circ},-40^{\circ}\right],\left[29^{\circ}, 35^{\circ}\right]\right.$ and $\left[-55^{\circ},-48^{\circ}\right]$ for each of the first three joints respectively). These variations are due to the presence of backlash zones where the joints can move but the encoders provide no measurements. Since this system fuses the information from three different sensors, it is able to adapt the offsets estimates in order to compensate the lack of information from the encoders.

Starting the iCub head in four different configurations with a full reset of the encoders between each experiment, the proposed system was run, calibrating the joint offsets. After each experiment, the head was homed to the calibrated zero position and the recorded gravity vector readings are shown in table II.

We can see that the gravity vector is practically vertical
TABLE II

GRAVITY VECTOR COMPONENTS IN THE ZERO POSITION

\begin{tabular}{|c|c|c|c|}
\hline Exp. \# & $g_{x}\left(\mathrm{~m} / \mathrm{s}^{2}\right)$ & $g_{y}\left(\mathrm{~m} / \mathrm{s}^{2}\right)$ & $g_{z}\left(\mathrm{~m} / \mathrm{s}^{2}\right)$ \\
\hline 1 & -0.022 & -9.835 & -0.016 \\
2 & 0.034 & -9.844 & -0.096 \\
3 & -0.102 & -9.829 & -0.091 \\
4 & -0.051 & -9.851 & -0.010 \\
\hline
\end{tabular}

(with an accuracy of 99.99\%). This experiment shows that the system is able to converge to a solution which agrees with the absolute gravity readings when started in different head configurations.

\section{B. Repeatability analysis of the calibration procedure}

The correct behavior of the system is also validated by ensuring its repeatability. To test the repeatability of the calibration procedure, we ran the algorithm with the iCub head started in six different configurations without a full reset of the encoders, meaning the offsets were the same for all experiments. Figure 5 shows the convergence of the offset estimates in each experiment with the mean value taken in the last 500 iterations shown in table III along with the standard deviation. The results show that the different experiments converge to similar values thus empirically proving the robustness of the proposed method to different starting conditions.

TABLE III

REPEATABILITY - MEAN VALUES OF THE OFFSET COMPONENTS

\begin{tabular}{|c|c|c|c|c|c|c|}
\hline Exp.\# & $\delta_{0}\left(^{\circ}\right)$ & $\delta_{1}\left(^{\circ}\right)$ & $\delta_{2}\left(^{\circ}\right)$ & $\delta_{3}\left(^{\circ}\right)$ & $\delta_{4}\left(^{\circ}\right)$ & $\delta_{5}\left(^{\circ}\right)$ \\
\hline 1 & -43.8 & 31.7 & -50.2 & -2.1 & 37.3 & -42.4 \\
2 & -43.4 & 32.6 & -52.7 & -3.4 & 35.5 & -43.7 \\
3 & -43.4 & 31.4 & -50.9 & -1.5 & 36.9 & -40.4 \\
4 & -43.1 & 32.0 & -52.0 & -3.9 & 37.4 & -42.2 \\
5 & -43.4 & 32.8 & -51.1 & -2.3 & 39.8 & -42.1 \\
6 & -43.0 & 33.0 & -52.9 & -3.9 & 35.7 & -41.6 \\
\hline std. dev. & $0.28^{\circ}$ & $0.64^{\circ}$ & $1.07^{\circ}$ & $1.02^{\circ}$ & $1.54^{\circ}$ & $1.08^{\circ}$ \\
\hline
\end{tabular}
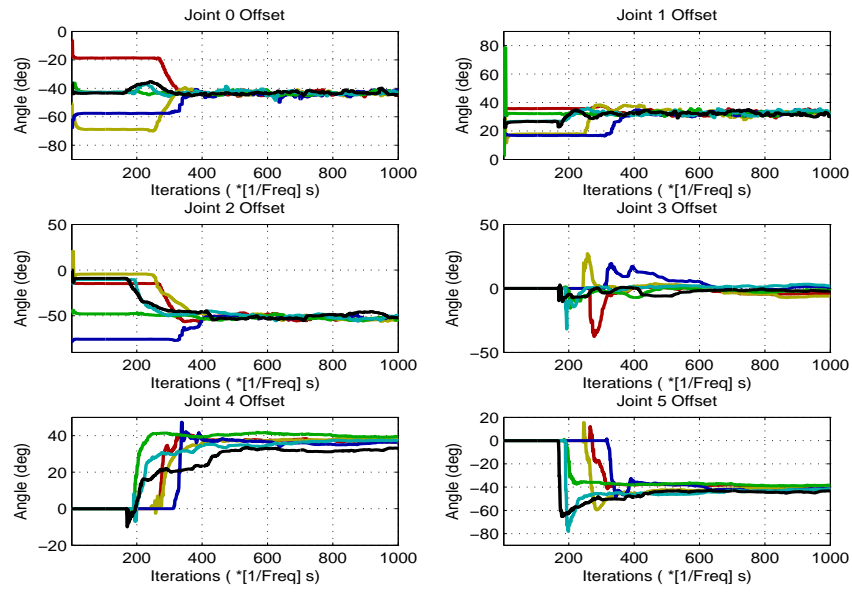

Fig. 5. Repeatability: convergence of the joints offsets.

\section{Generality of the calibration system}

As previously mentioned, the system was implemented in two other robotic platforms (Kobian and Vizzy) with 


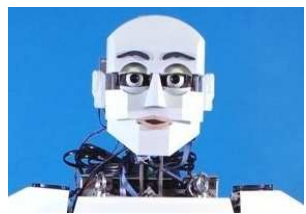

(a) Kobian head

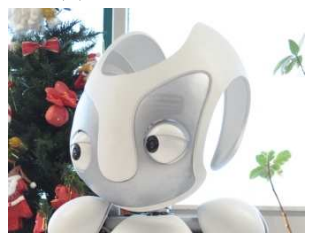

(b) Vizzy head

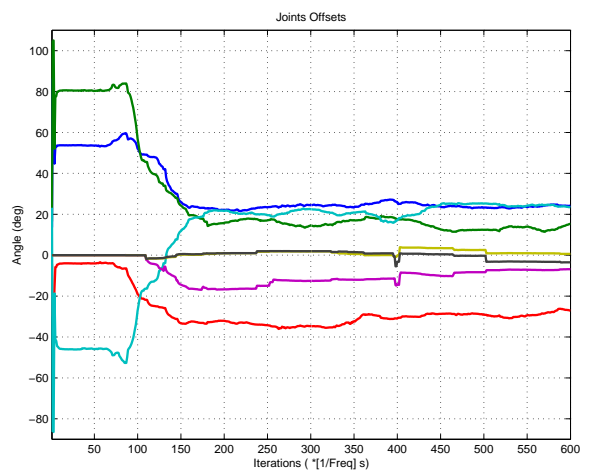

(c) Kobian, joint offsets (head moving)

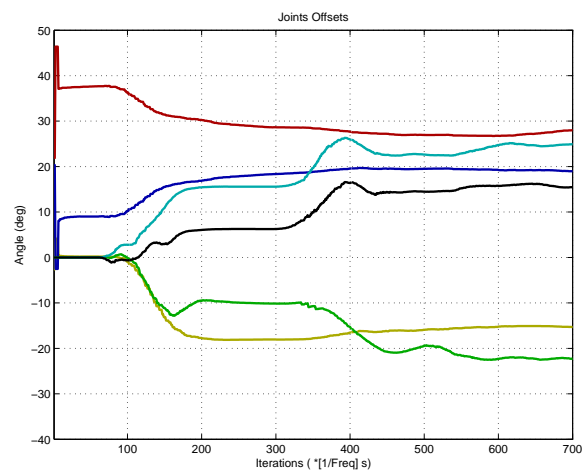

(d) Vizzy, joint offsets (head moving)

Fig. 6. Application of the online calibration procedure to other robot-heads, namely the Kobian head, Waseda University, (a), and the Vizzy head, IST/UTL University, (b). Results for the Kobian joint offsets, joints 0 till 6, are coded in colors blue, green, red, light blue, purple, yellow and black (c). Offsets estimated along time for the seven joints of the Kobian (c) and the six joints of the Vizzy (d).

different kinematic models from the iCub. The results for both calibrations are shown in figure 6(c) and (d).

The convergence of the system to the correct offsets values shows that the proposed online calibration methodology can be applied to different robotic platforms regardless of the kinematic chain, when equipped with either image or IMU.

\section{CONCLUSions}

We have presented a robust, online calibration system for the joints of a robot head. This calibration system is able to provide accurate estimates for the offsets of the joints, irrespective of the head initial configuration.

The approach uses information from the embedded inertial sensor, the relative encoders of the joints and vision and it performs robustly in the presence of noise and disturbances such as backlash in some joints. As opposed to other methors, our approach is very efficient and calibration can be achieved in a matter of a couple of seconds and a few movements of the robot head.

Our work provides a way of correctly initializing the robot, no matter what the robot's starting position might be, which is absolutely mandatory before the robot can engage in complex tasks. We present results with the iCub head, as humanoid robots represent the best metaphor of complex sensorimotor chains. To show the generality of the method, we have also applied the same methodology to other humanoid robots, such as the Kobian (from Waseda University) and Vizzy (IST-Lisbon), with different kinematic structures.

The relevance of this calibration procedure is that it allows the system to maintain an accurate internal model of its sensorimotor chains. These models are key for the control of humanoid robots, as the complexity of the tasks and diversity of the operational environments may be overwhelming. Instead, these models allow the system to contrast its observations to model-based predictions, substantially simplifying certain tasks, a mechanism similarl to the one presumably used by humans.

\section{REFERENCES}

[1] E. Datteri, G. Teti, C. Laschi, G. Tamburrini, P. Dario, and E. Guglielmelli, "Expected Perception: an anticipation-based perception-action scheme in robots," in IEEE/RSJ Int. Conf. on Intelligent Robots and Systems, Oct. 2003, pp. 934-939.

[2] _ - "Expected Perception in robots: a biologically driven perceptionaction scheme," in Proceedings of ICAR 2003, 11th International Conference on Advanced Robotics, vol. 3, 2003, pp. 1405-1410.

[3] C. Laschi, G. Asuni, E. Guglielmelli, G. Teti, R. Johansson, M. Carrozza, and P. Dario, "A bio-inspired neural sensoty-motor coordination scheme for robot reaching and preshaping," in Autonomous Robot, vol. 25, 2008, pp. 85-101.

[4] M. Hoffmann, H. Marques, A. H. Arieta, H. Sumioka, M. Lungarella, and R. Pfeifer, "Body schema in robotics: a review," IEEE Trans. Auton. Mental Develop., vol. 2, no. 4, pp. 304-324, December 2010.

[5] M. Hersch, E. L. Sauser, and A. Billard, "Online learning of the body schema," I. J. Humanoid Robotics, vol. 5, no. 2, pp. 161-181, 2008.

[6] R. Cantin-Martinez, M. Lopes, and L. Montesano, "Body schema acquisition through active learning," in IEEE International Conference on Robotics and Automation, Alaska, USA, 2010.

[7] B. Tworek, A. Bernardino, and J. Santos-Victor, "Visual selfcalibration of pan-tilt kinematics structures," Proc. of the 8th Conference on Autonomous Robot Systems and Competitions, ROBOTICA 2008, April 2008.

[8] J. Santos, A. Bernardino, and J. Santos-Victor, "Sensor-based selfcalibration of the icub's head," IEEE/RSJ International Conference on Intelligent Robots and Systems, IROS 2010, 2010.

[9] G. Broyden, "A class of methods for solving nonlinear simultaneous equations," in Mathematics of Computation, vol. 19, October 1965 , pp. $577-593$.

[10] S. Soatto, "A geometric framework for dynamic vision," $\mathrm{PhD}$ in Control and Dynamical Systems, California Institute of Technology, 1996.

[11] C. Harris and M. Stephens, "A combined corner and edge detector," The Plessey Company, pp. 147-152, 1988.

[12] R. Beira, M. Lopes, M. Praca, J. Santos-Victor, A. Bernardino, G. Metta, F. Becchi, and R. Saltarn, "Design of the robot-cub (icub) head," IEEE International Conference on Robotics and Automation, ICRA 2006, May 2006

[13] N. Endo, S. Momoki, M. Zecca, M. Saito, Y. Mizoguchi, K. Itoh, and A. Takanishi, "Development of whole-body emotion expression humanoid robot," IEEE Int. Conf. Robotics and Automation, ICRA 2008, pp. 2140-2145, 2008. 